\title{
Plasmapheresis in the treatment of multi-drug intoxication involving levothyroxine sodium and calcium channel blockers: a case report
}

\author{
Ran $\mathrm{Li}^{1 \wedge}$, Yong-Wei $\mathrm{Xu}^{2} \wedge$, Ying $\mathrm{Xue}^{1}$, Xian-Zheng $\mathrm{Wu}^{2}$ \\ ${ }^{1}$ Department of Endocrinology and Metabolism, Tongji Hospital, Tongji University School of Medicine, Shanghai, China; ${ }^{2}$ Department of \\ Emergency Medicine, Tongji Hospital, Tongji University School of Medicine, Shanghai, China \\ Correspondence to: Xian-Zheng Wu. Department of Emergency Medicine, Tongji Hospital, Tongji University School of Medicine, No. 389, Xincun \\ Road, Shanghai 200065, China. Email: wuxianzheng1962@163.com.
}

\begin{abstract}
Plasmapheresis, a procedure used to remove large molecular weight, protein-bound molecules from a patient's blood, has been shown to be useful in some cases of drug overdose. Levothyroxine sodium intoxication may result from the intentional or accidental ingestion of excessive amounts of the hormone, which can trigger a thyroid storm. However, case reports about the extremely large dose of $15,000 \mu \mathrm{g}$ of thyroxine intoxication are extremely rare, and even combined with calcium channel blockers (CCBs) poisonings. We present a case of an intentional poisoning with high doses of thyroxine, diltiazem and amlodipine successfully treated with plasma exchange. A 40-year-old woman was admitted showing unconsciousness and sustained hypotension with high levels of thyroid hormones (THs). It was discovered that she had secretly ingested at least 15,000 $\mu \mathrm{g}$ of levothyroxine sodium and CCBs with unknown amounts of diltiazem and amlodipine. Following plasmapheresis, the levels of TH declined dramatically after each of the 4 sessions, with hemodynamics gradually stabilizing and mental state improving. The early and timely use of plasmapheresis appears to be a vital therapeutic tool for the management of acute and severe forms of l-thyroxine and CCB intoxication. Its use can prevent thyroid storm and reverse the disturbances in the patient's hemodynamic status.
\end{abstract}

Keywords: Case report; levothyroxine sodium; calcium channel blockers (CCBs); intoxication; plasmapheresis

Submitted Jan 11, 2020. Accepted for publication Aug 14, 2020.

doi: $10.21037 / \mathrm{apm}-20-190$

View this article at: http://dx.doi.org/10.21037/apm-20-190

\section{Introduction}

Levothyroxine sodium tablet, $50 \mu \mathrm{g}$, has been marketed for decades (1) and has been used for the treatment of hypothyroidism and for the inhibition of thyroidstimulating hormone (TSH) levels in patients with thyroid nodules and/or thyroid gland enlargement (2). However, this medicine has a narrow therapeutic index and may put patients at risk of iatrogenic hyperthyroidism or hypothyroidism if the dose is only $25 \%$ lower or $25 \%$ higher than the optimal dose, depended on the patient's TSH concentration (3). Meanwhile, calcium channel blockers (CCBs) also have a narrow therapeutic index. The clinical symptoms of poisoning patients may include hypotension, bradycardia pulmonary edema (4).

At present, the current clinical rescue methods to treat patients with drug poisoning is to remove the toxic substances not yet absorbed from the gastrointestinal

^ ORCID: Ran Li, 0000-0003-2304-6282; Yong-Wei Xu, 0000-0002-7416-4281. 
tract. Depending on the situation, the first main treatment methods include vomiting, gastric lavage, activated carbon adsorption, magnesium sulfate catharsis, or mannitol catharsis. At the same time, blood and excreta (urine, feces, vomit) specimens can be collected for toxicological identification. Then, the function of each important organ can be assessed and given active, supportive treatment. The use of blood purification methods is considered for removing toxic substances absorbed into the body, depending on the severity of the disease. At the same time, as soon as it is possible to identify the source and type of toxic substances, the particular antidote for symptomatic treatment is applied. When taking an overdose of levothyroxine tablets and CCBs together, a series of toxic symptoms will result, such as hypotensive shock, arrhythmia, consciousness disorder, etc. $(2,4)$. Once thyroid storm and circulatory system failure occur, the mortality rate of patients is as high as $25 \%$ (5). For drug intoxication, plasmapheresis can rapidly and effectively remove toxic substances and their potentially toxic metabolites from the blood compartment, especially those with high proteinbinding. As the potential benefit of therapeutic plasma exchange is increasingly recognized, its use is becoming more widespread, and case reports have confirmed its value in the treatment of drug overdose (6). However, there is rare case report of plasma exchange successfully curing at least $15,000 \mu \mathrm{g}$ of extremely large levothyroxine sodium intoxication, and simultaneous poisoning with two different CCBs drugs. In this report, we describe a patient who overdosed on a combination of levothyroxine sodium and CCBs. The application of plasmapheresis dramatically reversed the severe biochemical and clinical manifestations and was able to prevent serious co-occurrence. We present the following case in accordance with the CARE reporting checklist, available at http://dx.doi.org/10.21037/apm-20190 (7).

\section{Case presentation}

All procedures performed in studies involving human participant were in accordance with the ethical standards of the institutional research committees and with the Helsinki Declaration (as revised in 2013). Written informed consent was obtained from the patient for publication of this manuscript and any accompanying images.

A 40-year-old woman was found unconscious by her husband at 5:00 am on September 15, 2019, with signs of dyspnea, vomiting, and empty bottles of medicine beside her. In an attempt to commit suicide, she had taken an excessive dose of drugs, including at least a 15,000 $\mathrm{\mu g}$ dose of sodium levothyroxine and unknown amounts of diltiazem and amlodipine. Her medical history included schizophrenia. When she was sent to the emergency department of a neighboring hospital at 10:00 am, she had a pulse of $75-85$ beats per minute, with a blood pressure of $83 / 43 \mathrm{mmHg}$ and an oxygen saturation level of $99 \%$. Gastric lavage was performed at once (the contents are unknown). A computed tomography (CT) scan of the brain revealed no abnormalities. Because the patient's blood pressure was still low, the patient was transferred to our hospital for further treatment at 13:00 pm. Physical examination revealed hypotension $(72 / 40 \mathrm{mmHg})$ and drowsiness, but no tachycardia and no enlargement of the nodules of the thyroid. Neurological examinations revealed no added abnormalities. The laboratory results are shown in Table 1. Biochemical analyses of plasma demonstrated that hepatic and renal functions were both within normal limits, whereas hyperthyroid function was above normal limits due to the oversuppletion of levothyroxine. These analyses yielded the following results: free thyroxine (fT4) level was $115.1 \mathrm{pmol} / \mathrm{L}$ [reference range (RR), 11.5-22.7 pmol/L]; free triiodothyronine (fT3) level was $11.55 \mathrm{pmol} / \mathrm{L}$ (RR, 3.5-6.5 pmol/L); total thyroxine (tT4) level was $387.0 \mathrm{nmol} / \mathrm{L}$ (RR, 58.1-140.6 $\mathrm{nmol} / \mathrm{L}$ ); total triiodothyronine (tT3) level was $3.19 \mathrm{nmol} / \mathrm{L}$ (RR, 0.92-2.79 nmol/L); TSH level was $0.15 \mathrm{mIU} / \mathrm{L}$ (RR, 0.3$5.5 \mathrm{mIU} / \mathrm{L}$ ); thyroglobulin antibody levels were $<20 \mathrm{IU} / \mathrm{mL}$ (RR, <60 IU/mL); thyroid peroxidase antibody levels were $<28.0 \mathrm{IU} / \mathrm{mL}(\mathrm{RR},<60 \mathrm{IU} / \mathrm{mL})$. As a result of these findings, levothyroxine sodium poisoning was determined.

Hemoperfusion was performed at 22:00 that day, and the patient gradually recovered consciousness. However, other symptoms did not improve, and norepinephrine was given to support blood pressure, while pantoprazole was given to protect the gastric mucosa.

The next day, September 16, 2019, the hemodynamic instability had not improved clinically. The progressive heart rate had increased to faster than before, fluctuating in 90-110 times/min, and the body temperature was $39.2^{\circ} \mathrm{C}$. Meanwhile, the toxicological identification agency test reported that the toxicants were diltiazem and amlodipine. However, the agency was unable to detect the levothyroxine sodium levels. Thus, we administered the first plasmapheresis (plasma volume 3,000 mL) removal, prescribed metoprolol (12.5 $\mathrm{mg}$ bid $\mathrm{PO}$ ) for controlling the heart rate, and propylthiouracil (PTU, $100 \mathrm{mg}$ tid PO) was 
Table 1 Auxiliary examination results of the patient

\begin{tabular}{lll}
\hline Examination items & Results & Normal value \\
\hline WBC $\left(\times 10^{9} / \mathrm{L}\right)$ & 8.90 & $3.5-9.5$ \\
$\mathrm{~N}\left(\times 10^{9} / \mathrm{L}\right)$ & 7.77 & $1.8-6.3$ \\
$\mathrm{~L}\left(\times 10^{9} / \mathrm{L}\right)$ & 0.55 & $1.1-3.2$ \\
$\mathrm{PLT}\left(\times 10^{9} / \mathrm{L}\right)$ & 239 & $125-350$ \\
$\mathrm{HB}(\mathrm{g} / \mathrm{L})$ & 108 & $115-150$ \\
$\mathrm{BNP}(\mathrm{pg} / \mathrm{mL})$ & 477.7 & $<100$ \\
$\mathrm{FBG}(\mathrm{mmol} / \mathrm{L})$ & 17.35 & $3.90-6.10$ \\
$\mathrm{~K}(\mathrm{mmol} / \mathrm{L})$ & 3.42 & $3.5-5.3$ \\
\hline
\end{tabular}

WBC, white blood cells; N, neutrophil; L, lymphocytes; PLT, platelet; HB, hemoglobin; BNP, B-type natriuretic peptide; FBG, fasting blood glucose; $\mathrm{K}$, serum potassium.

Table 2 Dynamic monitoring of thyroid indexes after treatment

\begin{tabular}{lllllllll}
\hline Thyroid indexes & $9.16^{\mathrm{a}}$ & $9.17^{\mathrm{b}}$ & $9.18^{\mathrm{c}}$ & $9.19^{\mathrm{d}}$ & $9.20^{\mathrm{e}}$ & 9.21 & 9.23 & 9.24 \\
\hline fT3 $(\mathrm{pmol} / \mathrm{L})$ & 11.55 & 9.57 & 8.04 & 6.41 & 7.69 & 5.97 & 6.04 & 6.05 \\
fT4 $(\mathrm{pmol} / \mathrm{L})$ & 115.10 & 104.13 & 99.71 & 59.94 & 41.12 & 31.75 & 30.49 & 30.14 \\
tT3 $(\mathrm{nmol} / \mathrm{L})$ & 3.19 & 2.33 & 2.02 & 2.02 & 1.95 & 2.02 & 2.04 & 1.57 \\
tT4 (nmol/L) & 387.0 & 355.4 & 349.2 & 282.8 & 253.6 & 196.5 & 191.5 & 166.8 \\
TSH (mlU/L) & 0.15 & 0.04 & 0.03 & 0.02 & 0.04 & 0.02 & 0.01 & 0.01 \\
\hline
\end{tabular}

${ }^{a}$, after Hemoperfusion; ${ }^{b}$, after the first plasmapheresis; ${ }^{c}$, after the second plasmapheresis; ${ }^{\mathrm{d}}$, after the third plasmapheresis; ${ }^{\mathrm{e}}$, after the fourth plasmapheresis.

given for reducing the conversion of $\mathrm{T} 3$ to $\mathrm{T} 4$.

On the third day, September 17, 2019, the patient's blood pressure returned to normal after the second plasma exchange; however, the patient showed signs of panhidrosis, and the body temperature was still febrile (Figure 1). Given the long half-life of the levothyroxine sodium and the fact that the patient's symptoms had not fully resolved, we continued with plasmapheresis treatment for the following 2 days and measured thyroid function each day. Laboratory data of thyroid function are shown in Table 2.

As seen in Table 2, thyroid function gradually improved, especially that of fT4. The dose of PTU (50 mg tid PO) was decreased on September 21, 2019, before discharge to avoid the inhibition of normal thyroid function. To date, the patient underwent PTU (50 mg tid PO) and metoprolol (12.5 $\mathrm{mg}$ bid PO) maintenance therapy, and was discharged on September 24, 2019, and was required to follow up after 2 weeks. After 2 weeks of telephone follow-up, the patient did not complain of special discomfort and was followed up at the local hospital, the entire pathogenesis is shown in
Figure 2.

\section{Discussion}

Thyroid hormones (THs) refer to thyroxine (T4) and triiodothyronine (T3), accounting for about $93 \%$ and $7 \%$ of the total respectively $(8,9)$. More than $99 \%$ of the circulating $\mathrm{T} 3$ and $\mathrm{T} 4$ is bound to plasma proteins, only about extremely small percentage of $\mathrm{tT} 3$ and $\mathrm{tT} 4$ is free $(10,11)$. The half-life of T4 is about 7 days, whereas that of T3 is one day (12). T3 mediates most of the hormonal actions because of its greater affinity for $\mathrm{TH}$ receptors whereas T4 acts mostly as a prohormone that becomes activated upon its conversion to T3. Release of THs from the thyroid gland is responded to TSH from pituitary, which in turn regulated by hypothalamic thyrotropinreleasing hormone (TRH). Therefore, TH exert negative feedback mechanisms through the hypothalamic-pituitarythyroid (HPT) axis (9).

Hyperthyroidism, also called thyrotoxicosis, is a 
condition that occurs due to excessive production and secretion of TH by the thyroid gland or leakage due to thyroid gland inflammation or excessive oral consumption of TH tablets (13). The clinical manifestations of patients with hyperthyroidism may range widely from asymptomatic forms to thyroid storm, according to the duration of illness, age of the patient, magnitude of hormone excess, symptoms are related to more organ systems by the TH's stimulation of catabolism, and enhancement of sensitivity to catecholamines, including tachycardia, cognitive dysfunction, tremor, fatigability and sweating (2). Thyroid storm is characterized by thermoregulatory dysfunction,

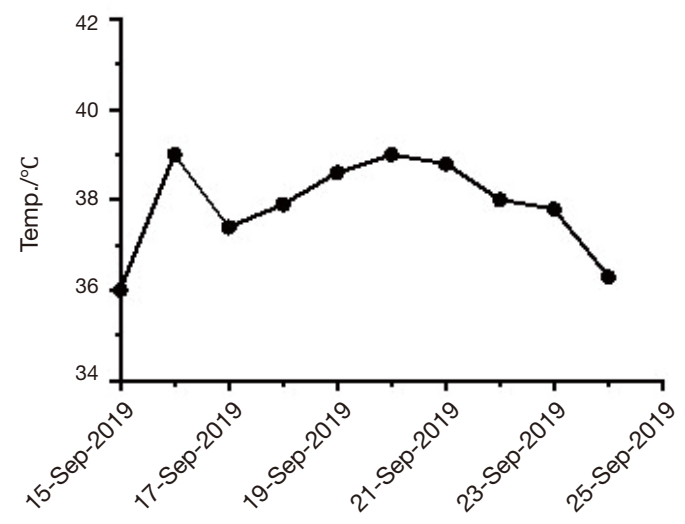

Figure 1 Trend and change of daily maximum temperature of the patient. tachycardia/atrial fibrillation, gastro-hepatic dysfunction, congestive heart failure as well as disturbances of consciousness. In our case, thyroid dysfunction developed following intentional ingestion of excessive doses of levothyroxine.

Levothyroxine sodium, biochemically and physiologically identical to endogenously produced $\mathrm{T} 4$, is a reliable and commonly prescribed drug to treat thyroid disease, but excessive dosage may bring about thyrotoxicosis (14). The gastrointestinal absorption of levothyroxine is approximately $80 \%$ (15). Therefore, when the patient was diagnosed as oral drug poisoning, the neighboring hospital quickly gave gastric lavage treatment to block the continued absorption of drugs in the gastrointestinal tract.

After ingestion of levothyroxine, serum T4 levels peak at 2 to 4 hours (16). The increase in serum T3 levels after levothyroxine administration is slow because of the time required for T4 to T3 conversion. Serum T3 concentrations are generally stable during maintenance levothyroxine therapy, unlike after administration of triiodothyronine itself (17). The goal of the treatment of thyrotoxicosis is to correct the hypermetabolic state with the fewest side effects and the lowest incidence of hypothyroidism. Beta blockers offer prompt relief of sympathomimetic activity of hyperthyroidism such as palpitations tremor, and heat intolerance. PTU, antithyroid drug, block new hormone synthesis. Meanwhile both drugs inhibit the peripheral conversion of T4 to T3 $(18,19)$.

Given the extremely high concentrations of $\mathrm{T} 4$,

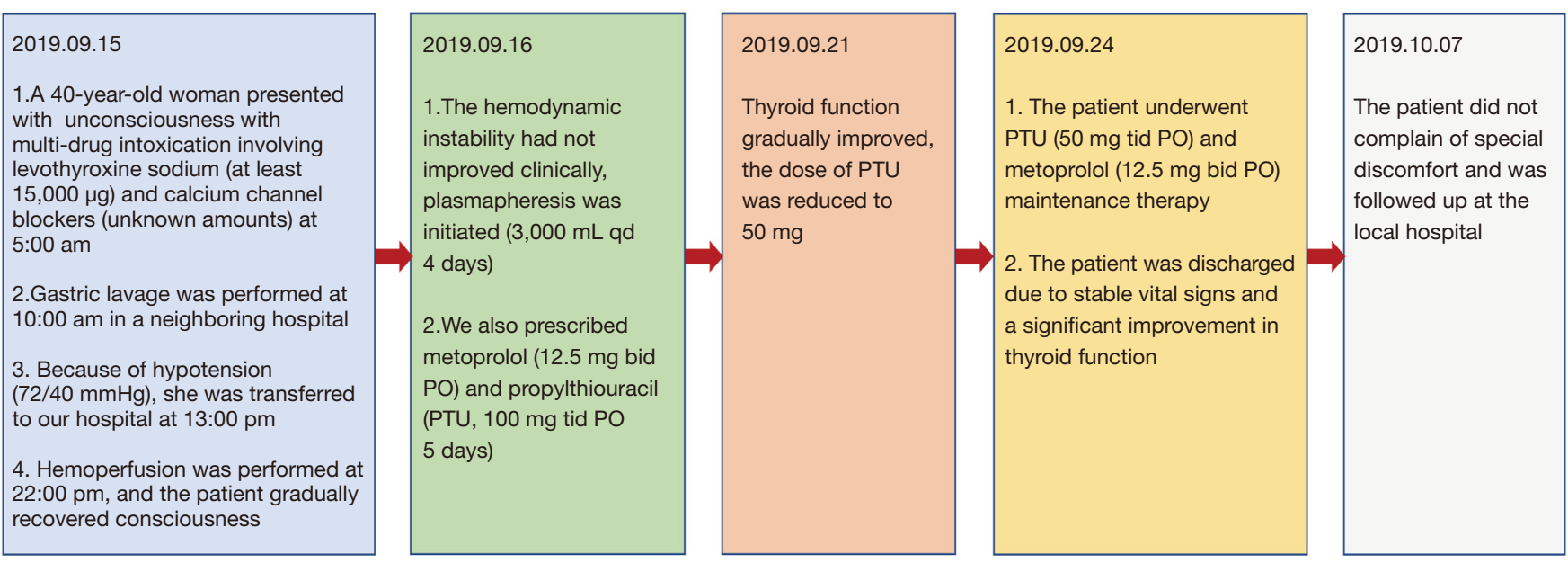

Figure 2 The summary of the patient's the whole process of treatment. 
treatment with drugs that inhibit the conversion of T4 to T3 may not clinically supply rapid relief, at least theoretically. Plasmapheresis can quickly and effectively restore thyroid function to normal for inpatients with hyperthyroidism. It is especially suitable for those patients who cannot take anti-thyroid drugs due to their side effects, and those who require normal thyroid function before emergency surgery. However, plasma exchange therapy cannot widely be used because it is expensive and invasive (20). Therefore, this treatment choice is only considered for patients that have severe intoxication, speculated high mortality, or a worsening condition despite conventional therapy (6). The patient with severe thyrotoxicosis due to thyroid storm have a high mortality rate. At first, we decided to remove circulating THs by hemoperfusion, because THs are firmly bound to plasma proteins. With the longer half-life of levothyroxine being an added potential risk (21), we decided that the patient should undergo plasmapheresis. Using this treatment, we observed dramatic improvements in clinical assessments and laboratory tests. Also, the patient did not develop complications of infection, allergic reaction, paresthesias, rigors, headache, or bleeding.

CCBs, a commonly used antihypertensive drug in clinical practice, directly block the entry of voltagegated L-type calcium channels inside the vascular smooth muscle and cardiac cells, weakening the excitation-contraction coupling (22). Therefore, the clinical effects in a patient with excessive intake may include sustained hypotension, atrioventricular conduction disturbances, bradycardia, pulmonary edema, and stroke, which are difficult to treat (23). Absorption of the drug begins within 30 to 60 minutes after oral administration (24), and immediate gastric lavage still works. Severe CCBs overdose can result in marked and sustained hypotension (25). Furthermore, CCBs have a high degree of protein binding (23). In our patient, the application of aggressive intravenous fluid resuscitation, vasopressors, and hemoperfusion failed to improve blood pressure.

Consequently, added plasmapheresis was performed the following day. Amlodipine, also known as dihydropyridine, causes hypotension and reflex tachycardia. Diltiazem, a drug that is not a dihydropyridine, can slow the heart rate (26). These results may account for the normal heart rate at the time of hypotension when the patient had initially taken a combination of amlodipine and diltiazem. Across the 4 sessions of plasmapheresis, serum levels of $\mathrm{tT} 3, \mathrm{tT} 4$, fT4, and fT3 decreased significantly. Plasmapheresis involves the clearing of protein-bound $\mathrm{TH}$; the colloids in the plasma provide new binding sites for $\mathrm{TH}$ that will be cleared during the next therapeutic plasma exchange (27). Binimelis et al. reported admitting 6 patients with erroneous massive intake of levothyroxine (70-1200 $\mathrm{mg}$ over an interval of 2-12 days) and concluded that plasmapheresis and hemoperfusion are both effective in removing $\mathrm{TH}$ from serum (28). However, in our case, the poor effect of plasma perfusion therapy may be due to the combined CCBs drug poisoning. Extraction in T4 was greater with plasmapheresis than with hemoperfusion. Although plasmapheresis is effective in ending circulating TH, it does not seem to be an effective way to accelerate the recovery of TSH from long-term inhibition (29).

In our case, the early application of plasma exchange successfully cured a variety of extremely large-dose drug poisonings that were rarely reported and reduced the occurrence of complications. However, after treatment, we only rechecked the patient's indicators and did not monitor the blood concentration. The change of blood drug concentration should be dynamically detected to evaluate the frequency of plasma exchange treatment to avoid drug residues and recurrence.

\section{Conclusions}

We present a case of a patient in a severe condition who overdosed on a combination of levothyroxine, diltiazem, and amlodipine. Our report further confirms that plasmapheresis can continuously improve the clinical status and laboratory examination index by rapid elimination of serum TH and CCB drugs. Doctors should consider the early use of therapeutic plasmapheresis after administering large amounts of high protein-binding drugs to lower the mortality rate; gastric lavage can also be performed without delay to block absorption of the drug.

\section{Acknowledgments}

Funding: This work was supported by the Key Developing Disciplines construction project of Shanghai Municipal Health Commission (number 2016ZB0204).

\section{Footnote}

Reporting Checklist: The authors have completed the CARE 
reporting checklist. Available at http://dx.doi.org/10.21037/ apm-20-190

Conflicts of Interest: All authors have completed the ICMJE uniform disclosure form (available at http://dx.doi. org/10.21037/apm-20-190). The authors have no conflicts of interest to declare.

Ethical Statement: The authors are accountable for all aspects of the work in ensuring that questions related to the accuracy or integrity of any part of the work are appropriately investigated and resolved. All procedures performed in studies involving human participant were in accordance with the ethical standards of the institutional research committees and with the Helsinki Declaration (as revised in 2013). Written informed consent was obtained from the patient for publication of this manuscript and any accompanying images.

Open Access Statement: This is an Open Access article distributed in accordance with the Creative Commons Attribution-NonCommercial-NoDerivs 4.0 International License (CC BY-NC-ND 4.0), which permits the noncommercial replication and distribution of the article with the strict proviso that no changes or edits are made and the original work is properly cited (including links to both the formal publication through the relevant DOI and the license). See: https://creativecommons.org/licenses/by-nc-nd/4.0/.

\section{References}

1. Patel H, Stalcup A, Dansereau R, et al. The effect of excipients on the stability of levothyroxine sodium pentahydrate tablets. Int J Pharm 2003;264:35-43.

2. Nguyen LT, Luong KV. Levothyroxine-induced hyperthyroidism. Allergy 2004;59:233-4.

3. Blakesley V, Awni W, Locke C, et al. Are bioequivalence studies of levothyroxine sodium formulations in euthyroid volunteers reliable? Thyroid 2004;14:191-200.

4. Kapelios CJ, Karamanakos G, Liatis S, et al. Recurrent episodes of life-threatening vasodilatory shock following unintentional intoxication with amlodipine. Hellenic J Cardiol 2017;58:369-71.

5. Swee S, Chng CL, Lim A, et al. Clinical characteristics and outcome of thyroid storm: a case series and review of neuropsychiatric derangements in thyrotoxicosis. Endocr Pract 2015;21:182-9.

6. Schutt RC, Ronco C, Rosner MH. The role of therapeutic plasma exchange in poisonings and intoxications. Semin Dial 2012;25:201-6.

7. Riley DS, Barber MS, Kienle GS, et al. CARE guidelines for case reports: explanation and elaboration document. J Clin Epidemiol 2017;89:218-35.

8. Goemann IM, Romitti M, Meyer ELS, et al. Role of thyroid hormones in the neoplastic process: an overview. Endocr Relat Cancer 2017;24:R367-85.

9. Helmreich DL, Parfitt DB, Lu XY, et al. Relation between the hypothalamic-pituitary-thyroid (HPT) axis and the hypothalamic-pituitary-adrenal (HPA) axis during repeated stress. Neuroendocrinology 2005;81:183-92.

10. Kaptein EM. Hormone-specific alterations of T4, T3, and reverse $\mathrm{T} 3$ metabolism with recent ethanol abstinence in humans. Am J Physiol 1997;272:E191-200.

11. Caro JF, Sloane NG, Glennon JA. Hyperthyroidism with normal serum total $\mathrm{T} 4, \mathrm{~T} 3$, and thyroid binding protein concentration. J Clin Pathol 1980;33:868-70.

12. Nicoloff JT, Low JC, Dussault JH, et al. Simultaneous measurement of thyroxine and triiodothyronine peripheral turnover kinetics in man. J Clin Invest 1972;51:473-83.

13. Ross DS, Burch HB, Cooper DS, et al. 2016 American Thyroid Association Guidelines for Diagnosis and Management of Hyperthyroidism and Other Causes of Thyrotoxicosis. Thyroid 2016;26:1343-421.

14. Mandel SJ, Brent GA, Larsen PR. Levothyroxine therapy in patients with thyroid disease. Ann Intern Med 1993;119:492-502.

15. Fish LH, Schwartz HL, Cavanaugh J, et al. Replacement dose, metabolism, and bioavailability of levothyroxine in the treatment of hypothyroidism. Role of triiodothyronine in pituitary feedback in humans. $\mathrm{N}$ Engl $\mathrm{J}$ Med 1987;316:764-70.

16. Browning MC, Bennet WM, Kirkaldy AJ, et al. Intraindividual variation of thyroxin, triiodothyronine, and thyrotropin in treated hypothyroid patients: implications for monitoring replacement therapy. Clin Chem 1988;34:696-9.

17. LeBoff MS, Kaplan MM, Silva JE, et al. Bioavailability of thyroid hormones from oral replacement preparations. Metabolism 1982;31:900-5.

18. Kravets I. Hyperthyroidism: Diagnosis and Treatment. Am Fam Physician 2016;93:363-70.

19. Guvenc B, Unsal C, Gurkan E, et al. Plasmapheresis in the treatment of hyperthyroidism associated with agranulocytosis: A case report. J Clin Apher 2004;19:148-50.

20. Ozdemir Baser O, Cetin Z, Catak M, et al. The 
role of therapeutic plasmapheresis in patients with hyperthyroidism. Transfus Apher Sci 2020;59:102744.

21. Koball S, Hickstein H, Gloger M, et al. Treatment of thyrotoxic crisis with plasmapheresis and single pass albumin dialysis: a case report. Artif Organs 2010;34:E5 5-8.

22. Graudins A, Lee HM, Druda D. Calcium channel antagonist and beta-blocker overdose: antidotes and adjunct therapies. Br J Clin Pharmacol 2016;81:453-61.

23. St-Onge M, Anseeuw K, Cantrell FL, et al. Experts Consensus Recommendations for the Management of Calcium Channel Blocker Poisoning in Adults. Crit Care Med 2017;45:e306-15.

24. Faulkner JK, McGibney D, Chasseaud LF, et al. The pharmacokinetics of amlodipine in healthy volunteers after single intravenous and oral doses and after 14 repeated oral doses given once daily. Br J Clin Pharmacol 1986;22:21-5.
25. Pichon N, François B, Chevreuil C, et al. Albumin dialysis: a new therapeutic alternative for severe diltiazem intoxication. Clin Toxicol (Phila) 2006;44:195-6.

26. Siddiqi TA, Hill J, Huckleberry Y, et al. Non-cardiogenic pulmonary edema and life-threatening shock due to calcium channel blocker overdose: a case report and clinical review. Respir Care 2014;59:e15-21.

27. Min SH, Phung A, Oh TJ, et al. Therapeutic Plasmapheresis Enabling Radioactive Iodine Treatment in a Patient with Thyrotoxicosis. J Korean Med Sci 2015;30:1531-4.

28. Binimelis J, Bassas L, Marruecos L, et al. Massive thyroxine intoxication: evaluation of plasma extraction. Intensive Care Med 1987;13:33-8.

29. Ezer A, Caliskan K, Parlakgumus A, et al. Preoperative therapeutic plasma exchange in patients with thyrotoxicosis. J Clin Apher 2009;24:111-4.
Cite this article as: $\mathrm{Li} \mathrm{R}, \mathrm{Xu} \mathrm{YW}, \mathrm{Xue} \mathrm{Y,} \mathrm{Wu} \mathrm{XZ.}$ Plasmapheresis in the treatment of multi-drug intoxication involving levothyroxine sodium and calcium channel blockers: a case report. Ann Palliat Med 2021;10(5):5839-5845. doi: 10.21037/ apm-20-190 